\title{
Model Predictive Control techniques for Hybrid Systems ${ }^{\text {th }}$
}

\author{
E.F. Camacho*, D.R. Ramírez, D. Limón, D. Muñoz de la Peña, T. Álamo \\ Dpto. Ingeniería de Sistemas y Automática, \\ Escuela Técnica Superior de Ingenieros, \\ Universidad de Sevilla, \\ Camino Descubrimientos, s/n., 41092 Sevilla
}

\begin{abstract}
This paper describes the main issues encountered when applying model predictive control to hybrid processes. Hybrid model predictive control (HMPC) is a research field non fully developed with many open challenges. The paper describes some of the techniques proposed by the research community to overcome the main problems encountered. Issues related to the stability and the solution of the optimization problem are also discussed. The paper ends by describing the results of a benchmark exercise in which several HMPC schemes were applied to a solar air conditioning plant.
\end{abstract}

Key words: Model predictive control, hybrid systems, solar refrigeration plant.

\section{Introduction}

Model predictive control (MPC) has been developed considerably in the last decades both in industry and in academia. This success is due to the fact that Model Predictive Control is perhaps the most general way of posing the control problem in the time domain. Model predictive control integrates optimal control, control of processes with constraints, with dead-time, multivariable processes and uses future references when available. The use of a finite horizon strategy allows the explicit handling of process and operational constraints by MPC. Although the technology originated in industry, the academic research community has contributed, during the last two decades, important results in all relevant aspects of the technique. It is widely accepted that the control of

\footnotetext{
A preliminary version of this paper was presented as a keynote paper of the plenary session at the 3rd IFAC Conference on Analysis and Design of Hybrid Systems (ADHS'09).

* Corresponding author

Email addresses: eduardo@esi.us.es (E.F. Camacho), danirr@cartuja.us.es (D.R. Ramírez), limon@cartuja.us.es (D. Limón), davidmps@cartuja.us.es (D. Muñoz de la Peña), alamo@cartuja.us.es (T. Álamo)
} 
linear processes with linear constraints (i.e. linear MPC) is a relatively mature research field. Linear models are good approximations if the process is kept close to an operating point and the nonlinearities are not too severe. However this is not the case of many processes where there are not only continuous variables but also variables that have a discrete nature.

For a long time, the control of processes with discrete variables and the control of processes with continuous variables were considered to be two completely different things. On the one hand, the theories of finite state machines were used to control processes with discrete variables, and on the other hand, linear and nonlinear control theory was used for the control of continuous variables. The techniques for modelling and analysis of these types of systems are different. In the case of continuous systems, differential equations, transfer functions, etc., are used as modelling tools, while in the discrete counterpart, state transition graphs, Petri Nets, etc., are employed. From the beginning of the 1990s there has been great interest in processes that have both discrete and continuous parts. Hybrid systems are dynamic systems with both continuous-state and discretestate and event variables. That is, the plant has time-driven and event-driven dynamics, the controller affects both time-driven and event-driven components, and it may deal with continuous and/or discrete signals.

The interest in hybrid systems has grown considerably during the last decade not only because it is a field relatively open to new advances but also because of their potential impact on the industrial applications of control. Hybrid systems arise naturally in several key areas such as automotive systems (Borrelli et al., 2006) or power systems (Ferrari-Trecate et al., 2004). A proof of this growing interest in hybrid systems is the number of sessions in major conferences and the existence of periodic conferences devoted exclusively to them.

This paper describes the main issues in analyzing and implementing MPC in the hybrid processes context. The paper starts with a short introductory review of the type of hybrid system models used in the MPC context. Then, the hybrid model implementation difficulties and some of the techniques proposed by the research community to overcome these problems are discussed. Issues related to computational burden and stability are also discussed. The paper ends by describing the results of a benchmark exercise in which several HMPC schemes were applied to a solar air conditioning plant.

\section{Hybrid Systems Description}

Hybrid systems are dynamic systems with both continuous-states, discretestates and event variables. That is, the plant has time-driven and event-driven dynamics, the controller affects both time-driven and event-driven components, and it may deal with continuous and/or discrete signals. Hybrid systems provide a good way to deal with plant-wide representation of complex industrial processes, which can be seen globally as hybrid systems due to the presence of subsystems and different working modes that commute along the plant operation. Also, there are some types of models that can be also seen as hybrid systems. For example, hierarchical systems can be modeled as hybrid systems 
in which lower level components are composed by continuous dynamical systems and higher level blocks are logic or decision modules (Antsalakis, 2000). Commuted systems (Sontag, 1981; Branicky, 1998; Johannson and Rantzer, 1998) can also be seen as hybrid models in which there exists a collection of dynamical systems and a set of commutation rules that can arise either from logical propositions or from a finite automaton such as a Petri net.

Different types of models can be used to describe hybrid systems. Hybrid modeling techniques used for control purposes have to be descriptive enough to capture the behavior of the various parts of the system; i.e., continuous dynamics (physical laws) and logic components (switches, automata, software code), and to take into account interconnections between logic and continuous dynamics. At the same time, the model has to be simple enough to solve analysis and synthesis problems. Although other models have been proposed (like fuzzy logic hybrid systems (Karer et al., 2008)), the most popular types of hybrid systems models used in MPC schemes are piecewise affine (PWA) systems and Mixed logical and dynamical (MLD) systems. Thus, this paper will be focused on these models.

PWA systems (Sontag, 1981) are amongst the most studied forms of hybrid systems. A PWA system is defined as

$$
\begin{gathered}
x(t+1)=A^{i} x(t)+B^{i} u(t)+f^{i} \\
y(t)=C^{i} x(t)+g^{i}
\end{gathered} \quad \text { for }\left[\begin{array}{l}
x(t) \\
u(t)
\end{array}\right] \in \mathcal{X}_{i}
$$

where $x(t) \in \mathbb{R}^{n}, u(t) \in \mathbb{R}^{m}$, and $y(t) \in \mathbb{R}^{r}$ denote the state, input, and output vectors, respectively. $\left\{\mathcal{X}_{i}\right\}_{i=1}^{s}$ is a polyhedral partition of the states and input space. Each $\mathcal{X}_{i}$ is given by

$$
\mathcal{X}_{i} \triangleq\left\{\left[\begin{array}{l}
x(t) \\
u(t)
\end{array}\right] \mid R^{i}\left[\begin{array}{l}
x(t) \\
u(t)
\end{array}\right] \leq r^{i}\right\}
$$

Each subsystem $\mathbb{S}^{i}$ defined by the 7-tuple $\left(A^{i}, B^{i}, C^{i}, f^{i}, g^{i}, R^{i}, r^{i}\right), i \in\{1,2, \ldots, s\}$, is termed as a component of the PWA system (1). $A^{i} \in \mathbb{R}^{n \times n}, B^{i} \in \mathbb{R}^{n \times m}$, and $\left(A^{i}, B^{i}\right)$ is a controllable pair. $C^{i} \in \mathbb{R}^{r \times n}$ and $R^{i} \in \mathbb{R}^{p_{i} \times(n+m)}$ and $f^{i}, g^{i}, r^{i}$ are suitable constant vectors. Note that $n$ is the number of states, $m$ is the number of inputs, $r$ is the number of outputs, and $p_{i}$ is the number of hyperplanes that define $\mathcal{X}_{i}$.

PWA systems are equivalent to sets of linear systems combined with finite automatons as pointed in Sontag (1996). On the other hand, PWA systems allow to model a wide class of processes such as linear systems with static piecewise affine nonlinearities or commuted systems. Moreover, they are able to approximate nonlinear dynamics arbitrarily well using local linearizations around different working points, and also they can approximate the more general type of nonlinear hybrid systems replacing the nonlinearities by piecewise affine approximations. They are also suitable for stability analysis and reachability analysis (Sontag, 1981).

Another interesting property of PWA systems is their equivalence to other types of hybrid systems models such as linear complementary systems, extended 
linear complementary systems, max-min-plus-scaling systems or the more popular mixed logical dynamical (MLD) systems (Heemels et al., 2001). All these models have their advantages, but the equivalence among all of them allow to transfer theoretical results from one type to other. And in this regard, PWA systems are by far the most studied of all of them.

The other type of hybrid system model that enjoys some popularity within the MPC community is the aforementioned mixed logical dynamical systems (MLD). They were proposed in Bemporad and Morari (1999) within the context of MPC techniques. In these systems there is a continuous part described by linear dynamics and a discrete part that it is modelled as a set of logical propositions. The key idea is that the set of logical propositions can be replaced by a set of equivalent linear constraints on binary integer (boolean) and continuous variables (Williams, 1993). The equivalence between propositions and mixed linear integer constraints is not a one-to-one relationship. Thus a single proposition could be replaced by a set of constraints. Although there is no systematic methodology to perform the substitution of every possible logic proposition, the most usual ones are tabulated (Mignone, 2001, 2002). Summing up, MLD systems are described by

$$
\begin{aligned}
x(t+1)= & A x(t)+B_{1} u(t)+B_{2} \delta(t)+B_{3} z(t) \\
y(t)= & C x(t)+D_{1} u(t)+D_{2} \delta(t)+D_{3} z(t) \\
& E_{1} x(t)+E_{2} u(t)+E_{3} \delta(t)+E_{4} z(t) \leq g
\end{aligned}
$$

where $x(t)=\left[\begin{array}{lll}x_{r}^{T}(t) & \left.x_{b}^{T}(t)\right]\end{array}\right]$ with $x_{r}(t) \in \mathbb{R}^{n}$ is the continuous part of the state vector and $x_{b}(t) \in\{0,1\}^{n_{b}}$ is the part of the state vector corresponding to the discrete part. Notice that if the state is discrete, not necessarily Boolean, but finite, it can be coded into a set of Boolean variables. The output signals also have a similar structure $y(t)=\left[y_{r}^{T}(t) y_{b}^{T}(t)\right]$ with $y_{r}(t) \in \mathbb{R}^{m}$ is the continuous part of the output and $y_{b}(t) \in\{0,1\}^{m_{b}}$ is the discrete part. The input vector $u(t)=\left[u_{r}^{T}(t) u_{b}^{T}(t)\right]$ is composed of a continuous part $u_{r}(t) \in \mathbb{R}^{l}$ and a discrete part $u_{b}(t) \in\{0,1\}^{l_{b}}$. Some auxiliary continuous $z(t) \in \mathbb{R}^{r}$ and discrete variables $\delta(t) \in\{0,1\}^{r_{b}}$ are usually needed. Obtaining the MLD description for a given hybrid system can be tedious and tricky. This task, however, can be eased by the use of a specialized software tool named HYSDEL (Torrisi and Bemporad, 2004).

\subsection{Identification and state estimation}

Since MPC techniques are based on a prediction model, it is clear that identification and state estimation methods are very important. Unfortunately identification and state estimation techniques for hybrid systems are less developed than their counterparts in other types of models such as linear systems. Most of the results and techniques are for PWA systems.

The problem of state observers for hybrid systems have been considered in the literature (Alessandri and Coletta, 2001b,a, 2003; Schinkel et al., 2003; Bara et al., 2000). It is usually assumed that the active mode of the system is known at every sampling time, although this is not always possible. When the 
active mode is not known, the estimation of the state becomes a harder problem that has been tackled for instance in Balluchi et al. (2002). In that work, it is proposed to use observers that takes as inputs not only the discrete inputs and outputs of the system but also discrete signals obtained from the continuous variables, that are measured to estimate the active mode. Then, the continuous state of the system can be observed using the techniques presented in Alessandri and Coletta (2001b,a, 2003); Bara et al. (2000). Observers designed using this procedure identify the active mode in finite time and the observation error is steered to a bounded set. A different approach is presented in Ferrari-Trecate et al. (2002), based on a moving horizon estimation. This strategy can be applied to PWA systems, but has a great computational burden that limits its real time implementation. Another approach that do not require knowing the active mode is that of Juloski et al. (2007). In that work, Luenberger observer design procedures are given for a class of bimodal piecewise linear (PWL) systems in both continuous and discrete time. On the other hand, it must be taken into account that if the state of a system is to be observed, then the system must be observable. For commuted and PWA systems there are precise definitions of observability (Bemporad et al., 2000). Also, a moving horizon estimation (MHE) technique has been presented in Pina and Botto (2006). The MHE method simultaneously estimates the state and the mode of the system and is based on a moving fixed-size estimation window which bounds the size of the optimization problem. This estimation technique deals with state estimation of MLD systems and can also estimate unknown inputs or disturbances acting on the hybrid system.

The other problem is hybrid systems identification. Most of the works consider Piecewise ARX (PWARX) models, which are a particular type of PWA models. A PWARX model can be obtained when the regressor space is divided into a finite set of polyhedral regions, and then obtaining an ARX system in each of them. Thus a PWARX is defined as:

$$
y(k)=f(x(k))+v(k)
$$

where $v(k)$ is a random noise,

$$
f(x)= \begin{cases}\theta_{1}^{T}\left[\begin{array}{l}
x \\
1
\end{array}\right] & \text { if } x \in \mathcal{X}_{1} \\
\vdots & \vdots \\
\theta_{s}^{T}\left[\begin{array}{l}
x \\
1
\end{array}\right] & \text { if } x \in \mathcal{X}_{s}\end{cases}
$$

is a PWA mapping and $x(k)$ is the regressor formed by measured values from the system input and outputs:

$$
x(k)=\left[y(k-1) \cdots y\left(k-n_{a}\right) u(k-1) \cdots u\left(k-n_{b}\right)\right]^{T}
$$

The identification of PWARX models consists in finding the parameters of the unknown PWA mapping $f$ from experimental data obtained from the plant. 
This can be considered as a piecewise affine regression problem. PWA regression is a problem that has been tackled in the neural network community (see Roll et al., 2004). Most of the methods deal only with continuous maps (Roll et al., 2004), although methods able to identify discontinuous mappings have been developed (Ferrari-Trecate et al., 2003; Bemporad et al., 2003; Vidal et al., 2003). One of the problems that arise in PWARX system identification is that of the sorting of the experimental data into each of the ARX subsystems that compose the PWARX model. Another problem is the identification of the polyhedral regions of the regressor space that contains the data sets that has been formed in the previous sorting phase. That later problem has been extensively treated in the pattern matching literature and can be solved using techniques such as supporting vectorial machines (SVM) (Vapnik, 1998) or the multi-category robust linear programming (MRLP) (Bennet and Mangasarian, 1994). The sorting problem is more difficult to solve, although there some techniques, often suboptimal, that have been proposed such as clustering techniques (Ferrari-Trecate et al., 2003), greedy algorithms (Bemporad et al., 2003) or algebraic procedures (Vidal et al., 2003). Another strategy based on a bayesian approach is presented in Juloski et al. (2005b), where the identification problem is posed as the problem of maximizing the total probability that the observed data is generated by the identified model. More recently, a structural approach for a class of hybrid systems which are linear and separable in the discrete variables has been recently presented in Nandola and Bhartiya (2009). This method (validated experimentally) identifies models corresponding to a certain number of modes, far fewer than the total possible modes of the system, generating the models for the remaining modes without any further requirement for input-output data by exploiting the separable structure of the hybrid system.

All these techniques have their drawbacks (Juloski et al., 2005a). The algebraic procedures often fails when there is a significant amount of noise in the data. The greedy algorithms involve the solution of NP-hard problems, thus they are not very useful. Moreover, sometimes they fail to assign a group to some regressors that have to be discarded, losing information. Also, clustering techniques such as Ferrari-Trecate et al. (2003) are promising, although they cannot use any a priori knowledge of the system. The bayesian approach (Juloski et al., 2005b) requires an a priori knowledge of the probability density functions of the unknown parameters of the system (although that knowledge does not need to be very precise provided that a sequential processing is used to improve the identification results). Finally, the approach presented in Nandola and Bhartiya (2009) requires an a priori knowledge of the system active mode.

\section{Model predictive control for hybrid systems}

Model predictive control (MPC) (Camacho and Bordóns, 2004) originated in the late seventies and has developed considerably since then. There are many applications of predictive control successfully in use at the current time, not only in the process industry but also applications to the control of other processes ranging from solar technology (Camacho et al., 1994) to flight control 
(Breger and How, 2006). Model Predictive Control is considered to be a mature technique for linear and rather slow systems like the ones usually encountered in the process industry, even though more complex systems, such as nonlinear, hybrid, or very fast processes, are not so amenable to the methods of MPC.

The term Model Predictive Control does not designate a specific control strategy but rather an ample range of control methods which make explicit use of a model of the process to obtain the control signal by minimizing an objective function over a finite receding horizon. In MPC the process model is used to predict the future plant outputs, based on past and current values and on the proposed optimal future control actions. These actions are calculated by the optimizer taking into account the cost function (where the future tracking error is considered) as well as the constraints.

Since this paper is focused on hybrid systems described as MLD or PWA system, in this section it is considered that the system described as the following model, which summarize both systems:

$$
\begin{aligned}
x(k+1)= & f(x(k), u(k), v(k)) \\
y(k)= & g(x(k), u(k), v(k)) \\
& h(x(k), u(k), v(k)) \leq 0
\end{aligned}
$$

where $x(k), u(k)$ and $y(k)$ are the state vector, manipulated variables and output vectors at instant $k . v(k)$ is a vector of auxiliary signals necessary to define the hybrid nature of the plant. These vectors may be split into a part which contains continuous variables and other with discrete (possibly binary) variables. If the model is described as a MLD system, the role of each signal can be directly inferred from equations (2)-(4) where $v=\left[z^{T}, \delta^{T}\right]^{T}$. If the model is described by a PWA, then the states and inputs are continuous and the auxiliary variable $v(k)$ is the integer index that define the partition where the state and input lie in. That is, the function $h(x, u, v)$ is defined as follows:

$$
h(x, u, v)=\mathcal{R}^{v}\left[\begin{array}{l}
x \\
u
\end{array}\right]-r^{v}, \text { where } v:\left[\begin{array}{l}
x \\
u
\end{array}\right] \in \mathcal{X}_{v}
$$

denoting the partition region as $\mathcal{X}_{v}=\left\{\left[x^{T}, u^{T}\right]^{T}: \mathcal{R}^{v}\left[x^{T}, u^{T}\right]^{T} \leq r^{v}\right\}$.

The states and inputs of the plant are constrained as follows

$$
\left[x(k)^{T}, u(k)^{T}\right]^{T} \in Z
$$

where $Z \in \mathbb{R}^{n+m}$ is a given closed set.

In order to use this system model to predict the future evolution of the plant from the current state $x$, a sequence of $N$ future inputs $\mathbf{u}=\{u(0), u(1), \cdots, u(N-$ $1)\}$ and auxiliary variables $\mathbf{v}=\{v(0), v(1), \cdots, v(N-1)\}$ must be known. The sequence of predicted states $\mathbf{x}=\{x, x(1), \cdots, x(N)\}$ and outputs $\mathbf{y}=$ $\{y(0), y(1), \cdots, y(N-1)\}$ can be obtained using recursively the model equation (5). Notice that not every pair $(\mathbf{u}, \mathbf{v})$ is admissible since they must fulfil $h(x(i), u(i), v(i)) \leq 0$ for all $i \in\{0,1, \cdots, N-1\}$.

From all the admissible pair $(\mathbf{u}, \mathbf{v})$, it is chosen the one which provides the best predicted evolution of the plant along a finite prediction horizon $N$. This is 
measured by a certain performance index which depends on the considered control objective. In the usual case of regulation to a given set-point or target $y_{s}, u_{s}$ and $v_{s}$, the performance index to be minimized penalizes the deviation between the predicted outputs, inputs, and auxiliary variables and their corresponding targets $y_{s}, u_{s}$ and $v_{s}$ respectively. This is typically posed as follows

$V_{N}(x, \mathbf{u}, \mathbf{v})=\sum_{\mathbf{i}=\mathbf{0}}^{\mathbf{N}-\mathbf{1}}\left\|\mathbf{y}(\mathbf{i})-\mathbf{y}_{\mathbf{s}}\right\|_{\mathbf{Q}}^{\mathbf{2}}+\left\|\mathbf{u}(\mathbf{i})-\mathbf{u}_{\mathbf{s}}\right\|_{\mathbf{R}}^{\mathbf{2}}+\left\|\mathbf{v}(\mathbf{i})-\mathbf{v}_{\mathbf{s}}\right\|_{\mathbf{T}}^{\mathbf{2}}+\mathbf{V}_{\mathbf{f}}\left(\mathbf{x}(\mathbf{N})-\mathbf{x}_{\mathbf{s}}\right)$

The function $V_{f}(x)$ is the so-called terminal cost function and it is added for stability reasons, as will be clarified in a next section.

Then, the optimal input sequence is derived from the solution of the following optimization problem $P_{N}(x)$ :

$$
\begin{array}{cl}
\min _{\mathbf{u}, \mathbf{v}} & V_{N}(x, \mathbf{u}, \mathbf{v}) \\
\text { s.t. } & x(i+1)=f(x(i), u(i), v(i)) \\
& y(i)=g(x(i), u(i), v(i)) \\
& h(x(i), u(i), v(i)) \leq 0 \\
& {\left[x(i)^{T}, u(i)^{T}\right]^{T} \in Z} \\
& x(N) \in X_{f}
\end{array}
$$

where the region $X_{f}$ is the so-called terminal region which is added, together with the terminal cost function $V_{f}$, for stability reasons.

The control law is derived from the solution of the optimization problem by means of the so-called receding horizon technique: At each sampling time $k$, the optimization problem is solved for the current state $P_{N}(x(k))$, but only the control action corresponding to the current sampling time is applied, and hence the optimization problem is repeatedly solved. Thus the control law is given by:

$$
u(k)=\kappa_{N}(x(k))=u^{*}(0 ; x(k))
$$

Notice that the receding horizon control is the responsible that the optimal solution be applied in a closed-loop manner, providing robustness to the closedloop system.

One of the advantages of MPC is that robust control ideas can be easily incorporated. The key idea is to take into account disturbances and uncertainties about the process in an explicit manner and to design MPC to optimize the objective function for the worst situation of the disturbances/uncertainties (Camacho and Bordóns, 2004).

As mentioned in section 2, PWA are equivalent to MLD systems, although there are more theoretical results for PWA models, including attempts to build explicit solutions, or stabilizing design. For PWA, the solution to this problem will be composed of the optimal control sequence $\mathbf{u}^{*}$ and also a vector $\mathcal{I}^{*}$ that represents the optimal commutation sequence. This optimal commutation sequence tells in which of the affine subsystems is the process state at each sampling time of the prediction horizon. The solution of problem $P_{N}(x)$ involves 
the same complexity for MLD models than for PWA models and it can be solved using the same techniques.

Another variant of PWA MPC controllers are those that consider an infinite horizon. The advantages of this controllers are better stability properties and guaranteed feasibility (Mayne et al., 2000). A drawback, however, is the the existence of an optimal solution is not guaranteed if constraints are taken into account. PWA MPC controllers with infinite horizon have been developed for LTI systems with quadratic cost function and also for piecewise linear cost functions and hybrid systems (Baotić et al., 2006). However, the main interest (from a hybrid systems point of view) is that explicit solutions can be built for PWA MPC.

The application of MPC to hybrid systems subject to disturbances or uncertainties gives rise to uncertain optimization problems. Many robust MPC schemes are based on the min-max strategy originally proposed in Witsenhausen (1968), where the performance index due to the worst possible disturbance realization is minimized. In all cases, the resulting min-max optimization problems are computationally very demanding and, in general, it is common feeling the control laws are too conservative. There are few papers in the literature that deal with this class of problems, most of them in a PWA (Fiacchini et al., 2008; Zou and Li, 2007) or a switched system (Mhaskar et al., 2005) framework. In the literature there are works on PWA MPC like Thomas et al. (2006) in which a controller that guarantees feasibility under bounded disturbances is presented together with a fast suboptimal algorithm.

Stochastic MPC takes a different route to solve MPC problems under uncertainty. Disturbances are modeled as random variables and the expected value of the cost function is minimized. Stochastic programming (SP) is a special class of mathematical programming that involves optimization under uncertainty (see Birge and Louveaux, 1997; Kall and Wallace, 1994; Ross, 1983). The original applications were agricultural economics, aircraft route planning and production of heating oil back in the 50's. Nowadays SP is becoming a mature theory that is successfully applied in several other application domains (see the survey Sahinidis, 2004). In particular, stochastic programming with integer variables has received a lot of attention lately (Schultz, 2003). Following this path, several stochastic MPC formulations for uncertain hybrid systems have been presented in van der Boom and Schutter (2001); Bemporad et al. (2006); Bemporad and Di Cairano (2006).

\section{Computational issues}

The MPC based on MLD models results in an optimization problem with a set of linear constraints and with real and integer (boolean in this case) decision variables. These types of optimization problems are known, in general, as mixed integer programming (MIP) problems. If the objective function is a linear function these problems are known as mixed integer linear programming (MILP) problems, or mixed integer quadratic programming (MIQP) problems when the objective function is quadratic. 
MILP or MIQP problems are much more difficult to solve than an LP or QP problems (see, for example, Floudas, 1995, for an excellent introduction), and some interesting properties like convexity are lost. Also, their complexity is of the NP-hard kind, and even to test if a feasible solution improves the best found so far is in itself an NP problem. Another drawback is the absence of optimality conditions, thus one can not known when a feasible solution is the optimal one.

A brute force approach to find the optimal solution would be to solve all of the QPs (LPs) that are associated to all the feasible combinations of the discrete decision variables. The solution will be the minimum of the solutions of all the QP (LP) problems. If all the discrete decision variables are Boolean, the number of possible QP (LP) problems is $2^{n_{b}}$. Fortunately there are more efficient ways of solving this type of problem. They are usually based on branch and bound methods (Fletcher and Leyffer, 1998) and solve only a portion of all QP (LP) problems. Some examples of solvers include GLPK (Makhorin, 2004) (public domain), Cplex (ILOG, Inc., 2004) (commercial), or Xpress-MP (Dash Associates, 2004) (commercial), for which Matlab interfaces are available.

The structure of the MPC problem and the reachability analysis tools have been used in Peña et al. (2003) to reduce the number of QP problems to be solved and for determining lower bounds to be used by branch and bounds algorithms in Peña et al. (2005). Besides branch and bound methods, other solution methods have been investigated to implement MPC controllers. In Bemporad and Giorgetti (2006) a solver that takes advantage of constraint satisfaction problems (CSP) solvers for dealing with satisfiability of logic constraints very efficiently was proposed. Also, heuristic approaches, although suboptimal in nature, can provide feasible solutions that can be admissible from a control point of view. Among these methods, genetic algorithms (Thomas et al., 2005; Olaru et al., 2004; Duzinkiewicz et al., 2009; Causa et al., 2008; Cortés et al., 2009) and ant colony algorithms (Sandou and Olaru, 2007) have been used.

On the other hand, another approach could be a modification to the MLD paradigm such as in Thomas et al. (2004), in which it is considered a partition of the state space in which, in turn, simpler MLD systems are defined. The results is a multi-MLD model that results in a MIQP problem that can be solved with lower computational burden. However, even with a reduced MIQP problem, the computational burden is still a problem that limits MLD MPC techniques to slow dynamics or short prediction horizons. Explicit implementations allow also to deal with the poor performance and/or feasibility problem that may appear when a stabilizing design is used (Morari and Baric, 2006). This technique allows to analyze stability and feasibility of the controller and also have a much lower computational burden. The attempts to find explicit solutions for hybrid MPC controllers have been more successful when a PWA model is considered.

When PWA predictions models are used, it can be seen that for a fixed commutation sequence, the resulting optimization problem $P_{N}(x)$ reduces to a QP or LP problem. In this case an explicit solution can be found using any of the methods available for linear systems (Gal and Nedoma, 1972; Acevedo and Pistikopoulos, 1997; Dua and Pistikopoulos, 2000; Tøndel et al., 2001; Bemporad 
et al., 2002b,a). The same can be said for every possible commutation sequence, thus to obtain the explicit solution for problem a multiparametric QP or LP problem must be solved for each possible commutation sequence. The optimal control sequence for a given process state $x$ will be obtained from the explicit solution of the multiparametric problem that gives a lower optimal cost (Borrelli, 2003). The resulting control law will be PWA, thus the closed loop system is also PWA. If the cost function is linear the state space partition is polyhedral but if a quadratic cost function is used the partition will not be polyhedral in a general case.

It is obvious that the biggest drawback of the previous strategy to find the explicit solution is the computational burden that grows exponentially with the prediction horizon. Most of the published works exploit the problem structure to compute the explicit solution as fast as possible. There are works in which the explicit solution is based on the hybrid model and use the iterative solution of multiparametric MILP and LP problems (Dua and Pistikopoulos, 2000). Other are based in dynamic programming and decompose the original problem in a series of multiparametric MILP or MIQP problems that are solved backwards recursively using the optimality principle of Bellman (Bellman, 1961). Examples of works that use this strategy include Kerrigan and Mayne (2002); Borrelli et al. (2005); Baotić et al. (2006).

Other problem with explicit implementations is the complexity of the state space partition, which grows exponentially with the dimension of the state and the prediction and control horizons. This happens even with linear systems and it is worse for hybrid systems. As the partition grows, the searching times and storage requirements can be too high for a practical implementation. Some works that help with this problems have been presented. Efficient techniques to evaluate the control signal for a given state $x$ have been presented in Johansen and Grancharova (2003); Tøndel et al. (2003). Other approach is to consider controllers with lower complexity, like a robust minimum time controller (Mayne and Schroeder, 1997) (analogous to a dead-beat controller but with guaranteed robust stability). A robust minimum time controller for hybrid systems (PWA) with guaranteed feasibility and stability under additive disturbances was presented in Grieder et al. (2005). The algorithm is based on a stabilizing controller together with the maximum admissible invariant set of the system, which works as a terminal constraint. The controller is designed solving backwards recursively $N$ MPC problems with a prediction horizon equal to 1 . Other algorithm is presented in Grieder and Morari (2003) where it is proposed a PWA control law that keeps the state contained in the maximum robust control invariant set, so that feasibility and stability under additive disturbances are guaranteed. A drawback, however, is that convergence to a region smaller than the maximum robust control invariant set is not guaranteed.

\section{Stabilizing model predictive control}

Predictive controllers belongs to the class of optimal control techniques since the control action to be applied minimizes a certain predicted performance cri- 
terion. The first difference with other optimal techniques is the finite prediction horizon, which allows to pose the problem as a mathematical programming problem to be solved at each sampling time. The second difference is the receding horizon policy used to derive the control law making the control action equals to the optimal solution corresponding to the current time. However, in general the optimal nature of the predictive control law does not ensure the stability of the closed loop system. Then, additional ingredients on the controller set-up must be considered in order to ensure closed-loop stability. These ingredients are typically two (Mayne et al., 2000): (i) the terminal cost function: a term penalizing the terminal state (i.e. the predicted state at the end of the prediction horizon) to be added in the cost function and (ii) the terminal constraint: an inequality constraint on the terminal state to be added to the constraints in the optimization problem.

The stabilizing formulation of the predictive control considering (without loss of generality) a PWA model of the hybrid, is shown in what follows. First, the cost function is given by

$$
V_{N}(x, \mathbf{u})=\sum_{j=0}^{N-1}\left\|x(j)-x_{s}\right\|_{Q}^{p}+\left\|u(j)-u_{s}\right\|_{R}^{p}+V_{f}\left(x(N)-x_{s}\right)
$$

where $\left(x_{s}, u_{s}\right)$ defines the target steady state and input. The optimization problem $P_{N}(x)$ minimizes this cost under the constraints imposed by the dynamics of the system and the limits on the states and inputs. Then $P_{N}(x)$ can be posed as follows:

$$
\begin{array}{ll}
\min _{\mathbf{u}} & J(x, \mathbf{u}) \\
\text { s.t. } & i(k) \in\left\{i:\left[\begin{array}{l}
x(k) \\
u(k)
\end{array}\right] \in \mathcal{X}_{i}\right\} \\
& x(k+1)=A^{i(k)} x(k)+B^{i(k)} u(k)+f^{i(k)}, \\
& {\left[x(j)^{T}, u(j)^{T}\right]^{T} \in Z, j \in\{0, \cdots, N-1\}} \\
& x(N) \in X_{f}
\end{array}
$$

The region of states where this optimization problem is feasible is denoted as $D_{N}\left(X_{f}\right)$. This region depends on the dynamics, the constraints, the length of the prediction horizon and the terminal set, and can be read as the set of states that can be steered to the terminal set in $N$ steps fulfilling the constraints.

As it is demonstrated in Mayne et al. (2000); Bemporad and Morari (2000), closed-loop stability can be ensured if: (i) the terminal region $X_{f}$ satisfies that for a given auxiliary control law $u=\kappa_{f}(x)$, for all $x \in X_{f},\left(x, \kappa_{f}(x)\right) \in Z$ and $x^{+}=A^{i} x+B^{i} \kappa_{f}(x)+f^{i} \in X_{f}$, with $i \in\left\{i:\left(x, \kappa_{f}(x)\right) \in \mathcal{X}_{i}\right\}$ and (ii) $V_{f}(x)$ is a definite positive function such that

$$
V_{f}\left(x^{+}\right)-V_{f}(x) \leq-\left\|x-x_{s}\right\|_{Q}^{p}-\left\|u(0)-u_{s}\right\|_{R}^{p}
$$


Under these conditions, the optimal cost function is a Lyapunov function for the closed-loop system and the feasibility region $D_{N}\left(X_{f}\right)$ is an invariant domain of attraction. In the case that the set of constraints is a polygonal region, the domain of attraction is a also a polygon that can be calculated by well-known methods (Kerrigan, 2000; Christophersen et al., 2004)

A remarkable property of this result is that the asymptotic stability property holds in the case of discontinuous model functions as those of the hybrid systems and in the case of non-convex and/or disconnected set of constraints.

Relaxed version of these properties have been proposed: in Limon et al. (2006) it is proved that the terminal constraint can be removed if the state is in a neighborhood of the set-point, and penalizing the terminal cost function, the MPC without terminal constraint is stabilizing in the interior of $D_{N}\left(X_{f}\right)$. Another interesting choice is the one proposed in Magni et al. (2001) where a prediction horizon larger than the control horizon is used deriving enhanced terminal conditions. In Limon et al. (2005) it is proved that using contractive terminal constraints, the terminal cost function calculation can be relaxed.

\subsection{Stabilizing design of $M P C$}

The design of a stabilizing MPC requires firstly defining the stage cost function (i.e. the $p$ norm and the weighting matrices $Q$ and $R$ ) and choosing the prediction horizon $N$ and then calculating off-line suitable terminal ingredients $\kappa_{f}(x), X_{f}$ and $V_{f}$. The complexity of the calculation of the terminal ingredients depends on the system to be controlled, the set of constraints and the chosen stage cost function. State-of-the-art procedures may be computationally demanding but since this is done off-line, this issue is not limiting. In the sequel, it will be assumed that the sets $\mathcal{X}_{i}$ and $Z$ are polygons, that is, union of convex polyhedra.

A simple method to accomplish the stabilizing design is choosing an equality terminal constraint

$$
\begin{aligned}
x(N) & =x_{s} \\
V_{f}(x) & =0
\end{aligned}
$$

The simplicity of this design is attractive, but the performance of the closed loop system and the domain of attraction can be improved by means of a more involved design.

There exists a number of techniques to calculate a suitable Lyapunov function and invariant region for hybrid systems (see for instance the survey Biswas et al., 2005, and references there in) and these can be used to compute the stabilizing terminal conditions. Among them, two main techniques are typically used (Mignone et al., 2000; Grieder et al., 2005; Lazar et al., 2006): LMI-based techniques and polyhedral techniques. The LMI-based method is used to calculate a suitable Lyapunov function to be used as terminal cost function. Besides, these methods can provide a suitable terminal region as a level curve of the calculated Lyapunov function. On the other hand, polyhedral methods are used 
to calculate invariant sets, though it can be used to calculate induced Lyapunov functions (Kerrigan, 2000).

The most simple approach consists in using techniques for linear systems. In effect if the target steady state and input is in the interior of a defining region $X_{i}$, then the system can be transformed in a linear systems by means a suitable change of variables. Considering the defining region as a hard constraint, a quadratic terminal cost function for the unconstrained system is calculated and the maximal admissible positively invariant set calculated by means of standard algorithms (Gilbert and Tan, 1991).

In case of considering larger regions, the hybrid dynamics of the system must be considered. In this case a quadratic Lyapunov function $V_{f}(x)=x^{T} P x$ and a piecewise linear control law $u=K_{i} x$ if $\left(x, K_{i} x\right) \in \mathcal{X}_{i}$ can be chosen. Sufficient conditions to calculate the Lyapunov matrix and the gains of the control law can be posed as LMIs. This result is improved if piecewise quadratic Lyapunov function $V_{f}(x)=x^{T} P_{i} x$ for $\left(x, K_{i} x\right) \in X_{i}$ is chosen as a Lyapunov function. A LMI-based sufficient condition has also been proposed (Grieder et al., 2005; Lazar et al., 2006). In these contributions methods for the computation of the terminal cost function in case of stage cost based on $1 / \infty$ norms.

The terminal region can be computed by using polyhedral methods. Given that the control law is piecewise linear, the closed-loop system is a piecewise affine autonomous system subject to polygonal constraints. An iterative method is used to calculate the maximal admissible positive invariant set. Since this method is finitely determined thanks to the contractiveness of the Lyapunov function, the terminal region is a polygon (Rakovic, 2005). A single polyhedral invariant set can be calculated by means of the method proposed in Alamo et al. (2008). This leads to a simpler region at expense of conservativeness.

\section{Applications of hybrid MPC}

Applications of hybrid MPC can be found in different fields. In automotive systems, hybrid MPC has been applied to traction control systems (Borrelli et al., 2006), and to adaptive cruise control (Corona et al., 2006b,a; Corona and De Schutter, 2008) (the latter work compares different controllers based on pure PWA models and PWA obtained from MLD descriptions). Also in power systems, there have been applications of hybrid predictive control to cogeneration plants (Ferrari-Trecate et al., 2004) or current converter control (Beccuti et al., 2007; Bâja et al., 2007; Geyer et al., 2008). More specific applications include the hybrid control of supermarket display cases (Larsen et al., 2005; Sarabia et al., 2009), the real-time optimization of public transport systems operations (Cortés et al., 2009) or the temperature control of a batch reactor (Karer et al., 2007; Causa et al., 2008). In all of these applications, there are several operation modes in the plant that justify the implementation of a hybrid MPC controller. In this paper we will consider in more detail a distinct application, the hybrid MPC control of a solar air conditioning plant. 


\subsection{Solar air conditioning plant}

The main problem encountered when controlling a solar energy process is that the primary source of energy cannot be manipulated and from a control point of view, the solar radiation acts as a perturbation. The control research community has contributed considerably in this field by designing advanced controllers for solar processes (Camacho et al., 1997),(Camacho et al., 1997; Garcia-Gabin et al., 2009).

The plant dealt with in here is a real solar refrigeration plant of certain complexity. The solar air conditioning plant (figure 1) is used to cool the laboratories of the Systems Engineering and Automation Department of the University of Seville. It consists of a solar field that produces hot water which feeds an absorption machine which generates chilled water. The chilled water is injected into the air conditioning system, achieving a cooling power of $35 \mathrm{~kW}$. A description and modelling can be found in Zambrano et al. (2008). A general scheme of the plant is presented in figure 2, showing its main components: the solar subsystem, composed of a set of flat solar collectors, the accumulation subsystem, composed of two tanks storing hot water, and the cooling subsystem. There also exists an auxiliary energy subsystem composed of a gas-fired heater that can supply energy in those situations where solar radiation is not enough, and a thermal load subsystem composed of a heat pump that allows to perform tests for different load profiles. The hybrid nature of the plant comes from the use of two different energy sources (solar and gas), which can be combined or used independently. Besides, thermal energy coming from a storage tank can be added to the system. The plant can be re-configured on-line manipulating open/close valves and ON/OFF pumps to allow selecting the components for energy supply.

The primary source of energy is the solar radiation, which is used by the solar collectors to increase the temperature of the circulating water. The solar field is composed of $151 \mathrm{~m}^{2}$ of flat collectors which work in the range of $60{ }^{\circ} \mathrm{C}$ to $100{ }^{\circ} \mathrm{C}$ and supply a nominal power of $50 \mathrm{~kW}$.

The hot water can be stored in two tanks of $2500 \mathrm{l}$ each and be used in transient situations where solar radiation is not sufficient. A gas-fired heater of $68 \mathrm{~kW}$ can be used to supplement the solar energy when necessary. The existing heater has a built-in $\mathrm{ON} / \mathrm{OFF}$ controller, which makes its outlet temperature rather oscillatory.

The cooling subsystem is composed of an absorption machine that works with water as cooling fluid and a water solution of lithium bromide $\left(\mathrm{H}_{2} \mathrm{O}-\mathrm{Li}-\mathrm{Br}\right)$. The machine requires that its inlet temperature is inside the range of $75-100{ }^{\circ} \mathrm{C}$ for chilled water production. The machine has four different circuits: evaporator, generator, condenser, and absorber; where the energy exchanges for chilled water production take place. A refrigeration tower is used to evacuate the heat.

A heat exchanger and a heat pump of $54 \mathrm{~kW}$ is used to absorbe the production of cold air. The subsystem has a PI controller to regulate the output temperature of the primary circuit of the heat exchanger. This value can be used to fix several profiles of the evaporator input temperature. 


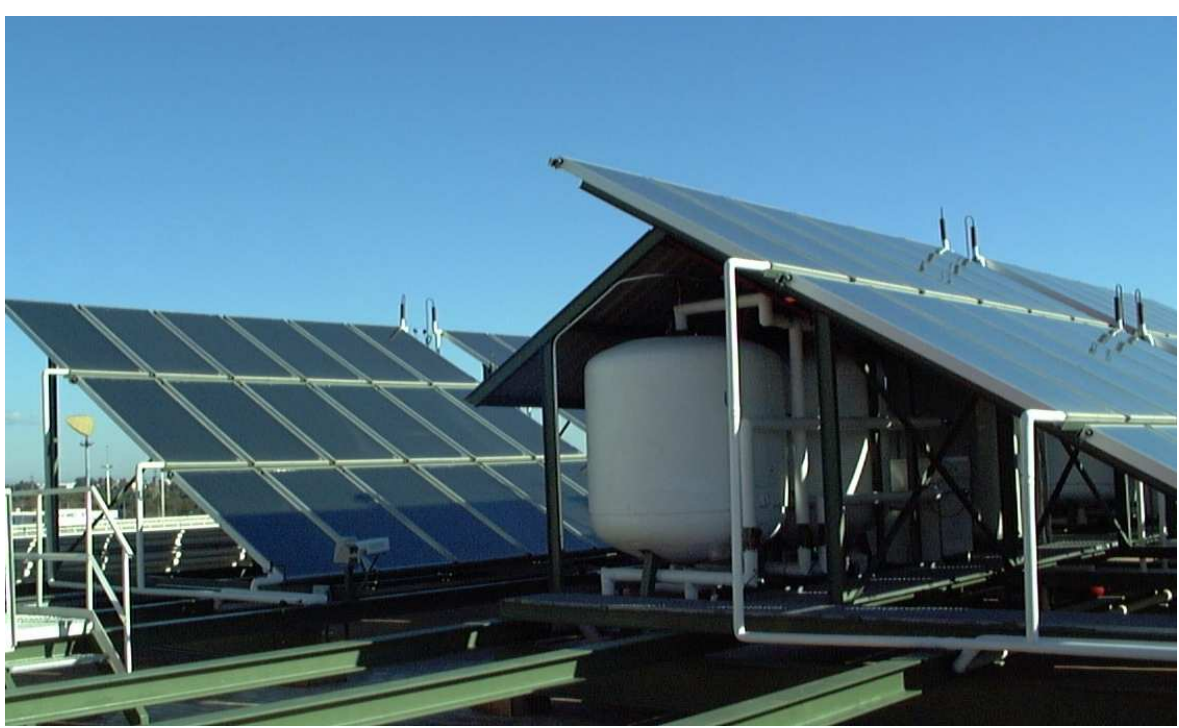

Figure 1: The solar air conditioning plant

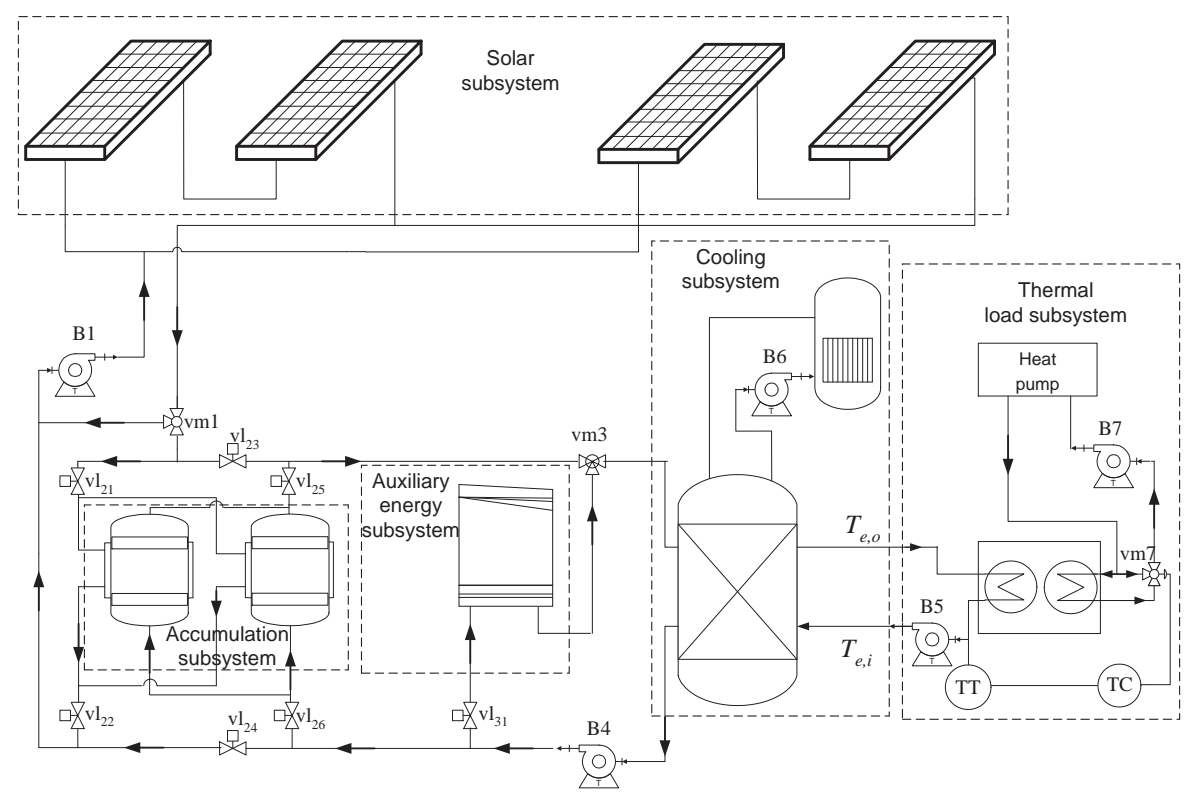

Figure 2: Scheme of Solar air conditioning plant 
The main manipulated variables are the field pump velocity and the mix valve position and a series of open/close valves and ON/OFF pumps which allow to change the operating modes of the plant described below.

There are temperature sensors for the solar collectors inlet and output water, tanks inlet and output, gas heater inlet, generator inlet and output temperatures and evaporator output temperatures. Flows can be measured at different places such as the generator and condenser of the absorption machine, the gas heater.

The system allows to measure disturbances such as environmental temperature, flow through the evaporator of the absorption machine, evaporator input temperature and solar radiation.

The plant evolves among several operating modes through of its reconfiguration during its daily operation. There are many operating possibilities but only 13 operating modes make sense:

1. Recirculation. All water flow through the solar collectors. In this mode, the water temperature may be increased.

2. Loading the tanks with hot water. The water in the solar collectors flows through the accumulators.

3. Using the solar collectors only. The water is heated in the solar collectors. The water flows to the absorption machine.

4. Using the solar collectors and gas heater. The water is heated in the solar collectors and the gas heater. The gas heater is used when the absorption machine input temperature is inadequate.

5. Using the gas heater, only. The water is heated in the gas heater, and then it flows through of the absorption machine.

6. Using the tanks and gas heater. The water of absorption machine is given by the accumulators. The gas heater is used when the absorption machine input temperature is not enough.

7. Using the tanks only. When the solar radiation is low, then the heat stored in the accumulators is used to operate the absorption machine.

8. Loading the tanks and using the gas heater. The accumulators are loaded with heated water by the solar collectors. The water of absorption machine is given by the gas heater.

9. Recirculation and using the gas heater. The water is recirculated through the solar collectors. The water goes to the absorption machine from the gas heater only.

10. Using the solar collectors and loading the tanks. The water from the solar collectors is divided into the tanks and the absorption machine.

11. Using the solar collectors and the gas heater, and loading the tanks. The water from the solar collectors is divided into the tanks and the absorption machine. The inlet water to the absorption machine comes from the solar collectors and gas heater.

12. Using the tanks and the gas heater to feed the absorption machine, and recirculation in the solar collectors. The water recirculates into the solar collectors. The water of generator comes from the accumulators and the gas heater. 
13. Using the tanks to feed the absorption machine, and recirculation in the solar collectors. The water from the accumulators circulates through the generator circuit. The water recirculates into the solar collectors.

The plant can operate under two general conditions: cooling demand exists or not exists. In the first case, the main control objective is to supply chilled water to the air distribution system according to the demanded temperature, and this implies to keep the absorption machine working. The absorption machine must be fed with hot water between $75^{\circ} \mathrm{C}$ and $100^{\circ} \mathrm{C}$. The water must be warmed using the solar collectors, the heat stored in the tanks, the gas heater or by an optimum combination of all these methods. The auxiliary energy (gas) consumption must be minimized because of environmental and economical reasons. Also, other control objective is to store as much energy as possible in the accumulators at the end day, because the plant works on a daily basis. Besides, the startup time must be minimized. In the second case, the control objective is to storage as much energy as possible in the tanks if the environmental conditions allow it.

The plant was used as a benchmark within the network of excellence HYCON funded by the European Commission under FP6. The benchmark exercise (Zambrano et al., 2006) consisted in comparing the results obtained by each controller under simulation and the results of the controller working for one day with the real plant. The simulation results had to be obtained for two days with the given environmental conditions corresponding to a clear day followed by a day with scattered clouds. The following quantities were to be measured: mean square error of evaporator temperature tracking, energy consumed by the gas heater, value of the tank temperature at the end of each day.

Different MPC approaches to the global operating control of this plant were presented for the benchmark exercise and four were selected to be published in an especial issue of the European Journal of control (Camacho, 2008) devoted to the benchmark exercise. The first approach can be seen in its extended version in Zambrano and Garcia-Gabin (2008), where a hierarchical scheme is presented. The higher level selects the operating mode based on an integer optimization problem with variable weights, and the lower level regulates the continuous variables using a set of MPC. The hybrid model considers the 13 operating modes and it is based on a MLD description. The extended version of the second approach was presented in Sonntag et al. (2008). The supervisory control scheme was designed from process insight gained from a thorough analysis of the energetic and dynamic aspects of the system. The discrete process inputs are adjusted by switching between a set of operating modes, and in each operating mode, the settings of the continuous inputs are chosen by a look-up table computed to minimize the consumption of auxiliary energy while ensuring a robust plant operation. The controller structure is based on a set of discrete supervisors, a continuous control algorithm and a set of emergency procedures. Only the first 8 operating modes are considered. The third approach was presented in Rodriguez et al. (2008) and it is based on a non-linear model predictive control strategy mixing control and economic aims that manipulates simultaneously 
the continuous and discrete elements and a set of logical rules corresponding to the hybrid nature of the control problem. The controller incorporates an internal model with embedded logic control and the concept of virtual control variables. This approach allows transforming the hybrid problem in a reduced order nonlinear model predictive control one, that can be solved with continuous optimization methods. The fourth approach (Menchinelli and Bemporad, 2008) proposes a multi-layer hybrid controller consisting of a high-level supervisor that decides the optimal operating mode on-line through a hybrid model predictive control strategy, a static lower-level controller defining proper set-points for the chosen mode, and existing standard low-level controllers that ensure robust tracking of such set-points. Although it provides a suboptimal solution, this hierachical approach preserves the basic hybrid structure of the problem and provides a satisfactory closed-loop performance. The control problem is divided in two steps. First, the hybrid MPC supervisor provides the optimal operating mode for the next time step, based on an abstracted model described in MLD form through the HYSDEL Programming Language. The currently most suitable operating configuration is computed by solving a relatively small-size MIQP problem on line. Then, the current values for the continuous variables are computed according to the chosen operating mode by exploiting control maps derived from experimental results, and tracked by the lower level controllers.

Some of the results obtained using the hierarchical scheme in (Zambrano and Garcia-Gabin, 2008) can be seen in figures 3 and 4 . Figure 3 shows the operating modes along the day and the solar radiation. It can be seen that the Hybrid MPC system decides the appropriate operating mode. Figure 4 shows the inlet and outlet temperature at the generator. Notice that the oscillations observed are due to the on-off gas heater, when the solar field produces enough energy, the behavior is smooth.

\section{Conclusions}

The main problems encountered when applying model predictive control to hybrid processes have been described in the paper. An application illustrating how HMPC can be applied in real time has been described showing that it is a technique that can be applied to real processes of moderate complexity. Hybrid model predictive control is a research field non fully developed with many open challenges. New types of hybrid systems can be considered in the MPC schemes, like the integral continuous-time hybrid automata recently presented in Cairano et al. (2009). There are not many strategies to solve certain problems like tracking in hybrid predictive controllers (Borrelli et al., 2009) and others are relatively open and active fields like hybrid systems identification, implementation techniques or new applications outside the industrial community. The authors are confident that further research in predictive control of hybrid systems will lead to a more mature predictive control technology able to cope with the real control problems that have to be faced in large scale or complex systems. 


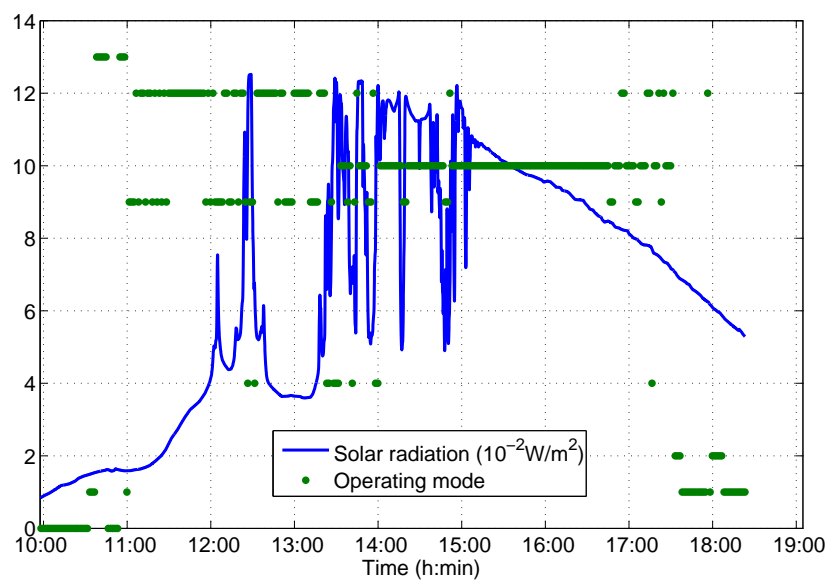

Figure 3: Operation modes of the solar air conditioning plant and solar radiation for an MPC experiment.

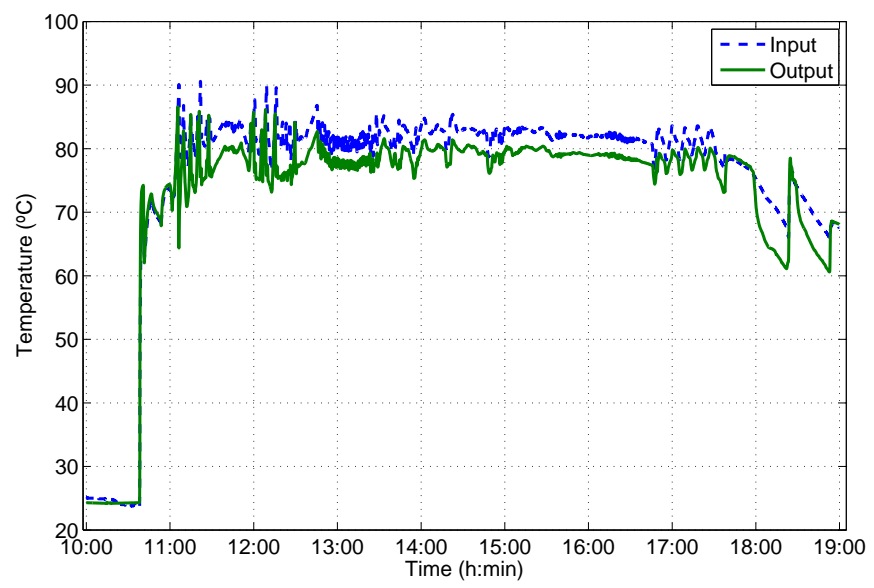

Figure 4: Inlet and outlet temperature at the generator of the solar air conditioning plant for the experiment of figure 3 . 


\section{Acknowledgement}

The authors would like to acknowledge the Spanish Ministerio de Educación y Ciencia for funding this work under grants DPI2007-66718-C04-01 and DPI2008-05818.

\section{References}

Acevedo, J., Pistikopoulos, E., 1997. A multiparametric programming approach for linear process engineering problems under uncertainty. Industrial Engineering Chemical Research 36, 717-728.

Alamo, T., Fiacchini, M., Cepeda, A., Limon, D., Bravo, J., Camacho, E., 2008. On the computation of robust control invariant sets for piecewise affine systems. In: R. Findeisen, F. A., Biegler, L. (Eds.), Assesment And Future Directions Of Nonlinear Model Predictive Control. No. 358 in Lecture Notes In Control And Information Series. pp. $131-139$.

Alessandri, A., Coletta, P., 2001a. A greedy approach to identification of piecewise affine models. In: Benedetto, M. D., Sangiovanni-Vincentelli, A. (Eds.), Hybrid Systems: Computation and Control 2001. Vol. 2034 of Lecture Notes in Computer Science. pp. 7-18.

Alessandri, A., Coletta, P., 2001b. Switching observers for continuous time and discrete time linear systems. In: Proc. of the 2001 American Control Conference. pp. 2516-2521.

Alessandri, A., Coletta, P., 2003. Design of observers for switched discrete time linear systems. In: Proc. of the 2003 American Control Conference. pp. 27852790 .

Antsalakis, P., 2000. A brief introduction to the theory and applications of hybrid systems. Proceedings IEEE, Special Issue on Hybrid Systems: Theory and Applications 88 (7), 879-886.

Bâja, M., Patiño, D., Cormerais, H., Riedinger, P., Buisson, J., 2007. Hybrid control of a three-level three-cell dc-dc converter. In: 2007 American Control Conference.

Balluchi, A., Benvenuti, L., Benedetto, M. D., Sangiovanni-Vincentelli, A., 2002. Design of observers for hybrid systems. In: Proc. of Hybrid Systems: Computation and Control 2002. pp. 76-89.

Baotić, M., Christophersen, F. J., Morari, M., Dec. 2006. Constrained Optimal Control of Hybrid Systems With a Linear Performance Index. IEEE Trans. on Automatic Control 51 (12), 1903-1919.

Bara, G., Daafouz, J., Kratz, F., Iung, C., 09 2000. State estimation for a class of hybrid systems. In: ADPM 2000. Shaker, pp. 313-316. 
Beccuti, A., G.Papafotiou, Morari, M., Almer, S., Fujioka, H., Jonsson, U., Kao, C., Wernrud, A., Rantzer, A., Bâja, M., Cormerais, H., Buisson, J., 2007. Hybrid control techniques applied to the step-up dc-dc converter. In: 2007 American Control Conference.

Bellman, R., 1961. Adaptive Control Processes - A guided tour. Princeton University Press.

Bemporad, A., Borrelli, F., Morari, M., 2002a. Model Predictive Control Based on Linear Programming - The Explicit Solution. IEEE Transactions on Automatic Control 47 (12), 1974-1984.

Bemporad, A., Di Cairano, S., 2006. Optimal control of discrete hybrid stochastic automata. Hybrid Systems: Computation and Control, Lecture Notes in Computer Science, 151-167.

Bemporad, A., Ferrari-Trecate, G., Morari, M., 2000. Observability and Controllability of Piecewise Affine and Hybrid Systems. IEEE Transactions on Automatic Control 45 (10), 1864-1876.

Bemporad, A., Garulli, A., Paoletti, S., Vicino, A., 2003. A greedy approach to identification of piecewise affine models. In: Maler, O., Pnueli, A. (Eds.), Hybrid Systems: Computation and Control 2003. Vol. 2623 of Lecture Notes in Computer Science. pp. 97-112.

Bemporad, A., Giorgetti, N., 2006. Logic-based solution methods for optimal control of hybrid systems. IEEE Transactions on Automatic Control 51, 963976.

Bemporad, A., Morari, F. B. M., 2000. Optimal controllers for hybrid systems: Stability and piecewise linear explicit form. In: Proceedings of the 39th IEEE Conference on Decision and Control.

Bemporad, A., Morari, M., 1999. Control of systems integrating logic, dynamics, and constraints. Automatica 35, 407-427.

Bemporad, A., Morari, M., Dua, V., Pistikopoulos, E., 2002b. The Explicit Linear Quadratic Regulator for Constrained Systems. Automatica 38, 3-20.

Bemporad, A., Muñoz de la Peña, D., Piazzesi, P., 2006. Optimal control of investments for quality of supply improvement in electrical energy distribution networks. Automatica 42, 1331-1336.

Bennet, K., Mangasarian, O., 1994. Multicategory discrimination via linear programming. Optimization Methods and Software 4, 27-39.

Birge, J., Louveaux, F., 1997. Introduction to Stochastic Programming. Springer, New York. 
Biswas, P., Grieder, P., Lofberg, J., Morari, M., 2005. A survey on stability analysis of discrete-time piecewise affine systems. In: Proceedings of the 16th IFAC World Congress.

Borrelli, F., 2003. Constrained Optimal Control of Linear \& Hybrid Systems. Vol. 290. Springer Verlag.

Borrelli, F., Baotic, M., Bemporad, A., Morari, M., 2005. Dynamic programming for constrained optimal control of discrete-time linear hybrid systems. Automatica 41, 1709-1721.

Borrelli, F., Bemporad, A., Fodor, M., Hrovat, D., 2006. An MPC/hybrid system approach to traction control. IEEE Transactions on Control Systems Technology 14 (3), 541-552.

Borrelli, F., Falcone, P., Pekar, J., Stewart, G., 2009. Reference governor for constrained piecewise affine systems. Journal of Process Control 19 (8), 1229 - 1237, special Section on Hybrid Systems: Modeling, Simulation and Optimization.

Branicky, M., 1998. Multiple lyapunov functions and other analysis tools for switched and hybrid systems. IEEE Transactions on Automatic Control 43, $475-482$.

Breger, L., How, J. P., 2006. Nonlinear model predictive control technique for unmaned air vehicles. Journal of Guidance, Control and Dynamics 29 (5), $1179-1188$.

Cairano, S. D., Bemporad, A., Júlvez, J., 2009. Event-driven optimization-based control of hybrid systems with integral continuous-time dynamics. Automatica 45 (5), $1243-1251$.

Camacho, E., 2008. Hybrid model predictive control of a solar refrigeration plant: Editorial of the special section. European Journal of Control 14 (6), 448-449.

Camacho, E., Berenguel, M., Bordons, C., 1994. Adaptive Generalized Predictive Control of a Distributed Collector Field. IEEE Trans. on Control Systems Technology 2, 462-468.

Camacho, E., Berenguel, M., Rubio, F., 1997. Advanced control of solar power plants. Springer Verlag, London.

Camacho, E., Bordóns, C., 2004. Model Predictive Control, 2nd Edition. Springer-Verlag.

Causa, J., Karer, G., Núñez, A., Sáez, D., Skrjanc, I., Zupancic, B., 2008. Hybrid fuzzy predictive control based on genetic algorithms for the temperature control of a batch reactor. Computers \& Chemical Engineering 32 (12), 3254 $-3263$. 
Christophersen, F., Baotic, M., Morari, M., 2004. Stability analysis of hybrid systems with a linear performance index. In: Proceedings of the 43rd IEEE Conference on Decision and Control.

Corona, D., De Schutter, B., 2008. Adaptive cruise control for a smart car: A comparison benchmark for mpc-pwa control methods. Control Systems Technology, IEEE Transactions on 16 (2), 365-372.

Corona, D., Lazar, M., De Schutter, B., Heemels, M., Oct. 2006a. A hybrid MPC approach to the design of a Smart adaptive cruise controller. In: Proceedings of the 2006 IEEE International Conference on Control Applications (CCA 2006). Munich, Germany, pp. 231-236.

Corona, D., Necoara, I., De Schutter, B., van den Boom, T., Dec. 2006b. Robust hybrid MPC applied to the design of an adaptive cruise controller for a road vehicle. In: Proceedings of the 45th IEEE Conference on Decision and Control. San Diego, California, pp. 1721-1726.

Cortés, C. E., Sáez, D., Milla, F., Núñez, A., Riquelme, M., 2009. Hybrid predictive control for real-time optimization of public transport systems' operations based on evolutionary multi-objective optimization. Transportation Research Part C: Emerging Technologies In Press, Corrected Proof, -.

Dash Associates, 2004. XPRESS-MP User Guide. http://www.dashoptimization.com.

Dua, V., Pistikopoulos, E., 2000. An algorithm for the solution of multiparametric mixed integer linear programming problems. Annals of Operations Research 99, 123-139.

Duzinkiewicz, K., Piotrowski, R., Brdys, M., Kurek, W., 2009. Genetic hybrid predictive controller for optimized dissolved-oxygen tracking at lower control level. Control Systems Technology, IEEE Transactions on 17 (5), 1183-1192.

Ferrari-Trecate, G., Gallestey, E., Letizia, P., Spedicato, M., Morari, M., 2004. Modeling and control of co-generation power plants: A hybrid system approach. IEEE Transactions on Control Systems Technology 12 (5), 694-705.

Ferrari-Trecate, G., Mignone, D., Morari, M., 2002. Moving horizon estimation for hybrid systems. IEEE Transactions on Automatic Control 47 (10), 1663 1676 .

Ferrari-Trecate, G., Muselli, M., Liberati, D., Morari, M., 2003. A clustering technique for the identification of piecewise affine systems. Automatica 39 (2), 205-217.

Fiacchini, M., Alamo, T., Alvarado, I., Camacho, E. F., 2008. Safety verification and adaptive model predictive control of the hybrid dynamics of a fuel cell system. International Journal of Adaptive Control and Signal Processing 22 (2), 142-160. 
Fletcher, R., Leyffer, S., 1998. Numerical experience with lower bounds for miqp branch-and-bound. SIAM Journal of Optimization 8 (2), 604-616.

Floudas, C., 1995. Non-Linear and Mixed Integer Optimization. Oxford Academic Press.

Gal, T., Nedoma, J., March 1972. Multiparametric Linear Programming. Management Science 18 (7), 406-422.

Garcia-Gabin, W., Zambrano, D., Camacho, E. F., 2009. Sliding mode predictive control of a solar air conditioning plant. Control Engineering Practice $17(6), 652-663$.

Geyer, T., Papafotiou, G., Morari, M., 2008. Hybrid model predictive control of the step-down dc-dc converter. Control Systems Technology, IEEE Transactions on 16 (6), 1112-1124.

Gilbert, E. G., Tan, K. T., 1991. Linear systems with state and control constraints: the theory and application of maximal output admissible sets. IEEE Transactions on Automatic Control AC-36, 1008-1020.

Grieder, P., Kvasnica, M., Baotic, M., Morari, M., 2005. Stabilizing low complexity feedback control of constrained piecewise affine systems. Automatica 41 , issue $10,1683-1694$.

Grieder, P., Morari, M., 2003. Complexity Reduction of Receding Horizon Control. In: IEEE Conference on Decision and Control. Maui, Hawaii, pp. 31793184 .

Heemels, W. P. M. H., Schutter, B. D., Bemporad, A., 2001. Equivalence of hybrid dynamical models. Automatica 37, 1085-1091.

ILOG, Inc., 2004. CPLEX 9.0 User Manual. Gentilly Cedex, France.

Johannson, M., Rantzer, A., 1998. Computation of piece-wise quadratic lyapunov functions for hybrid systems. IEEE Transactions on Automatic Control $43,555-559$.

Johansen, T., Grancharova, A., 2003. Approximate explicit constrained linear model predictive control via orthogonal search tree. IEEE Transactions on Automatic Control 48 (5), 810-815.

Juloski, A., Heemels, W., Ferrari-Trecate, G., Vidal, R., Niessen, J., Paoletti, S., 03 2005a. Comparison of four procedures for the identification of hybrid systems. In: HSCC 2005. Springer.

Juloski, A., Heemels, W., Weiland, S., 2007. Observer design for a class of piecewise linear systems. International Journal of Robust and Nonlinear Control 17 (15), $1387-1404$. 
Juloski, A., Weiland, S., Heemels, W., 2005b. A bayesian approach to identification of hybrid systems. IEEE Transactions on Automatic Control 50 (10), 1520 - 1533 .

Kall, P., Wallace, S., 1994. Stochastic Programming. Wiley, Chichester.

Karer, G., Music, G., Skrjanc, I., Zupancic, B., 2007. Hybrid fuzzy model-based predictive control of temperature in a batch reactor. Computers \& Chemical Engineering 31 (12), 1552 - 1564 .

Karer, G., Music, G., Skrjanc, I., Zupancic, B., 2008. Model predictive control of nonlinear hybrid systems with discrete inputs employing a hybrid fuzzy model. Nonlinear Analysis: Hybrid Systems 2 (2), 491 - 509.

Kerrigan, E., 2000. Robust Constraint Satisfaction: Invariant Sets and Predictive Control. Ph.D. thesis, University of Cambridge.

Kerrigan, E., Mayne, D., December, 10-13 2002. Optimal control of constrained, piecewise affine systems with bounded disturbances. In: Proc. 41th Conference on Decision and Control, CDC'2002.

Larsen, L., Geyer, T., Morari, M., July 2005. Hybrid model predictive control in supermarket refrigeration systems. In: Proceedings of 2005 IFAC World Congress. Praha, Czech Republic.

Lazar, M., Heemels, W. P. M. H., Weiland, S., Bemporad, A., 2006. Stabilizing model predictive control of hybrid systems. IEEE TRANSACTIONS ON AUTOMATIC CONTROL 51, 1813-1819.

Limon, D., Alamo, T., Camacho, E., 2005. Enlarging the domain of attraction of mpc controllers. Automatica 41, 629-635.

Limon, D., Alamo, T., Salas, F., Camacho, E., 2006. On the stability of constrained MPC without terminal constraint. IEEE Transactions on Automatic Control 51, 832-836.

Magni, L., De Nicolao, G., Magnani, L., Scattolini, R., 2001. A stabilizing model-based predictive control for nonlinear systems. Automatica 37, 13511362 .

Makhorin, A., 2004. GLPK (GNU Linear Programming Kit) User's Guide. URL http://www.gnu.org/software/glpk/glpk.html

Mayne, D., Rawlings, J., Rao, C., Scokaert, P., 2000. Constrained model predictive control: Stability and optimality. Automatica 36, 789-814.

Mayne, D., Schroeder, W. R., 1997. Robust time-optimal control of constrained linear systems. Automatica 33, 2103-2118.

Menchinelli, P., Bemporad, A., 2008. Hybrid model predictive control of a solar air conditioning plant. European Journal of Control 14 (6), 501-15. 
Mhaskar, P., H., E.-F. N., D., C. P., 2005. Robust hybrid predictive control of nonlinear systems. Automatica 41 (2), 209-217.

Mignone, D., 2001. The REALLY BIG Collection of Logic Propositions and Linear Inequalities. Tech. rep.

URL http://control.ee.ethz.ch/index.cgi?page=

publications; action=details;id=377

Mignone, D., Jan. 2002. Control and Estimation of Hybrid Systems with Mathematical Optimization. Ph.D. thesis.

URL http://control.ee.ethz.ch/index.cgi?page=

publications; action=details;id=11

Mignone, D., Ferrari-Trecate, G., Morari, M., 2000. Stability and stabilization of piecewise affine and hybrid systems: An lmi approach. In: Proceedings of the 39th IEEE Conference on Decision and Control.

Morari, M., Baric, M., 2006. Recent developments in the control of constrained hybrid systems. Computers \& Chemical Engineering 30 (10-12), 1619-1631.

Nandola, N. N., Bhartiya, S., 2009. Hybrid system identification using a structural approach and its model based control: An experimental validation. Nonlinear Analysis: Hybrid Systems 3 (2), 87 - 100.

Olaru, S., Thomas, J., Dumur, D., Buisson, J., 2004. Genetic algorithm based model predictive control for hybrid systems under a modified mld form. International Journal on Hybrid Systems 4 (1-2).

Peña, M., Camacho, E., Piñon, S., Carelli, R., July 2005. Model predictive controller for piecewise affine system. In: Proceedings of 2005 IFAC World Congress. Praha, Czech Republic.

Peña, M., Camacho, E. F., Piñon, S., June 2003. Hybrid systems for solving mpc of piecewise affine system. In: Proc. of the IFAC Conference on Analysis and Design of Hybrid Systems ADHS03.

Pina, L., Botto, M. A., 2006. Simultaneous state and input estimation of hybrid systems with unknown inputs. Automatica 42 (5), $755-762$.

Rakovic, S., 2005. Robust control of constrained discrete time systems: Characterization and implementation. Ph.D. thesis, Imperial College of Science, Technology and Medicine. Univ. of London.

Rodriguez, M., de Prada, C., Capraro, F., Cristea, S., 2008. Logic embedded nmpc of a solar air conditioning plant. European Journal of Control 14 (6), $484-500$.

Roll, J., Bemporad, A., Ljung, L., 2004. Identification of piecewise affine systems via mixed-integer programming. Automatica 40 (1), 37-50. 
Ross, S., 1983. Introduction to Stochastic Dynamic Programming. Academic Press, New York.

Sahinidis, N., 2004. Optimization under uncertainty: State-of-the-art and opportunities. Computers \& Chemical Engineering 28 (6-7), 971-983.

Sandou, G., Olaru, S., 2007. Ant colony and genetic algorithm for constrained predictive control of power systems. In: Bemporad, A., Bicchi, A., Buttazzo, G. (Eds.), Hybrid Systems: Computation and Control 2007. Vol. 4416 of Lecture Notes in Computer Science. pp. 501-514.

Sarabia, D., Capraro, F., Larsen, L. F., de Prada, C., 2009. Hybrid nmpc of supermarket display cases. Control Engineering Practice 17 (4), 428 - 441.

Schinkel, M., Heemels, W., Juloski, A., 2003. State estimation for systems with varying sampling rate. In: Proc. Conference on Decision and Control, CDC'03.

Schultz, R., 2003. Stochastic programming with integer variables. Mathematical Programming 97, 285-309.

Sonntag, C., Ding, H., Engell, S., 2008. Supervisory control of a solar air conditioning plant with hybrid dynamics. European Journal of Control 14 (6), 451-463.

Sontag, E., 1996. Interconnected automata and linear systems: A theoretical framework in discrete time. In: Alur, R., Henzinger, T., Sontag, E. (Eds.), Hybrid Systems III - Verification and Control. No. 1066 in Lecture Notes in Computer Science. pp. 436-448.

Sontag, E. D., April 1981. Nonlinear Regulation: The Piecewise Linear Approach. IEEE Trans. on Automatic Control 26 (2), 346-358.

Thomas, J., Dumur, D., Buisson, J., 2004. Predictive control of hybrid systems under a multi-mld formalism with state space polyhedral partition. In: ACC04 : American Control Conference. Boston (USA).

Thomas, J., Olaru, S., Buisson, J., Dumur, D., 2005. Genetic algorithm quadratic based predictive control for mld systems. In: 15th international Conference on control Systems and Computer Science. Bucarest.

Thomas, J., Olaru, S., Buisson, J., Dumur, D., 2006. Robust model predictive control for piecewise affine systems subject to bounded disturbances. In: Cassandras, C., Giua, A., Seatzu, C., Zaytoon, J. (Eds.), ADHS06: 2nd IFAC Conference on Analysis and Design of Hybrid Systems. Elsevier, Alghero (Italy), pp. 329-334.

Tøndel, P., Johansen, T., Bemporad, A., December, 4-7 2001. An Algorithm for Multi-Parametric Quadratic Programming and Explicit MPC Solutions. In: Proc. 40th Conference on Decision and Control, CDC'2001. 
Tøndel, P., Johansen, T. A., Bemporad, A., 2003. Evaluation of piecewise affine control via binary search tree. Automatica 39 (5), 945-950.

Torrisi, F., Bemporad, A., Mar. 2004. HYSDEL - A tool for generating computational hybrid models for analysis and synthesis problems. IEEE Transactions on Control System Technology 12, 235-249.

van der Boom, T., Schutter, B., 2001. Model predictive control for perturbed max-plus-linear stochastic systems: A stochastic approach. In: Proceedings of the 40th IEEE Conference on Decision and Control. Orlando, Florida USA, pp. $4535-4540$.

Vapnik, V., 1998. Statistical Learning Theory. John Wiley.

Vidal, R., Soatto, S., Sastry, Y. M. S., 2003. An algebraic geometric approach to the identification of a class of linear hybrid systems. In: Proc. Conference on Decision and Control, CDC'03.

Williams, H., 1993. Model Building in Mathematical Programming. John Wiley \& Sons, Third Edition.

Witsenhausen, H. S., 1968. A min-max control problem for sampled linear systems. IEEE Transactions on Automatic Control 13 (1), 5-21.

Zambrano, D., Bordons, C., Garcia-Gabin, W., Camacho, E., 2006. A solar cooling plant: a benchmark for hybrid systems control. In: the 2nd IFAC Conference on Analysis and Design of Hybrid Systems. pp. 199-204.

Zambrano, D., Bordons, C., Garcia-Gabin, W., Camacho, E. F., 2008. Model development and validation of a solar cooling plant. International Journal of Refrigeration 31, 315-327.

Zambrano, D., Garcia-Gabin, W., 2008. Hierarchical control of a hybrid solar air conditioning plant. European Journal of Control 14 (6), 464-483.

Zou, Y. Y., Li, S. Y., 2007. Robust model predictive control for piecewise affine systems. Circuits Systems and Signal Processing 26, 393-406. 